Supporting Information

For

\title{
A Colorimetric and Ratiometric Fluorescent Chemosensor with a Large Red-shift in Emission: Cu(II)-only Sensing by \\ Deprotonation of Secondary Amines as Receptor Conjugated to Naphthalimide Fluorophore
}

\author{
Zhaochao Xu, ${ }^{\dagger}$ Xuhong Qian,,$^{\dagger, *}$ and Jingnan Cui* ${ }^{\dagger}$ \\ ${ }^{\dagger}$ State Key Laboratory of Fine Chemicals, Dalian University of Technology, \\ Dalian 116012, China. \\ ${ }^{\ddagger}$ Shanghai Key Laboratory of Chemical Biology, School of Pharmacy, East \\ China University of Science and Technology, Shanghai 200237, China. \\ E-mail:xhqian@ecust.edu.cn orjncui@chem.dlut.edu.cn
}

Materials and Methods. All the solvents were of analytic grade and used as received. The solutions of metal ions were prepared from $\mathrm{LiClO}_{4} \cdot 3 \mathrm{H}_{2} \mathrm{O}, \mathrm{NaClO}_{4}, \mathrm{KClO}_{4}$, $\mathrm{MgCl}_{2} \cdot 6 \mathrm{H}_{2} \mathrm{O}, \mathrm{CaCl}_{2}, \mathrm{Fe}\left(\mathrm{NO}_{3}\right)_{3}, \mathrm{CoCl}_{2} \cdot 6 \mathrm{H}_{2} \mathrm{O}, \mathrm{NiCl}_{2} \cdot 6 \mathrm{H}_{2} \mathrm{O}, \mathrm{ZnCl}_{2}, \mathrm{CdCl}_{2} \cdot 2{ }_{2}{ }_{2} \mathrm{H}_{2} \mathrm{O}$, $\mathrm{CuCl}_{2} \cdot 2 \mathrm{H}_{2} \mathrm{O}, \mathrm{HgCl}_{2}, \mathrm{AgNO}_{3}, \mathrm{~Pb}\left(\mathrm{NO}_{3}\right)_{2}$, respectively, and were dissolved in distilled water. ${ }^{1} \mathrm{H}-\mathrm{NMR}$ were measured on a Bruker $\mathrm{AV}-400$ spectrometer with chemical shifts reported as ppm (in $\mathrm{CDCl}_{3} / \mathrm{DMSO}-\mathrm{d}_{6}$, TMS as internal standard). Mass spectra were measured on a HP 1100 LC-MS spectrometer. Melting points were determined by an X-6 micro-melting point apparatus and are uncorrected. IR spectra were recorded on a Nicolet Nexus 770 spectrometer. All pH measurements were made with a Sartorius basic pH-Meter PB-20. Fluorescence spectra were determined on a Hitachi F-4500. Absorption spectra were determined on a PGENERAL TU-1901 UV-Vis Spectrophotometer. The fluorescence quantum yields $\left(\Phi_{\mathrm{F}}\right)$ were estimated with $N$-butyl-4-butylamino-1,8-naphthalimide in absolute ethanol as a standard $\left(\Phi_{\mathrm{F}}=\right.$ $0.81){ }^{\text {S1 }}$ 


\section{Synthesis and Characteristics of Compounds 1 and 2.}

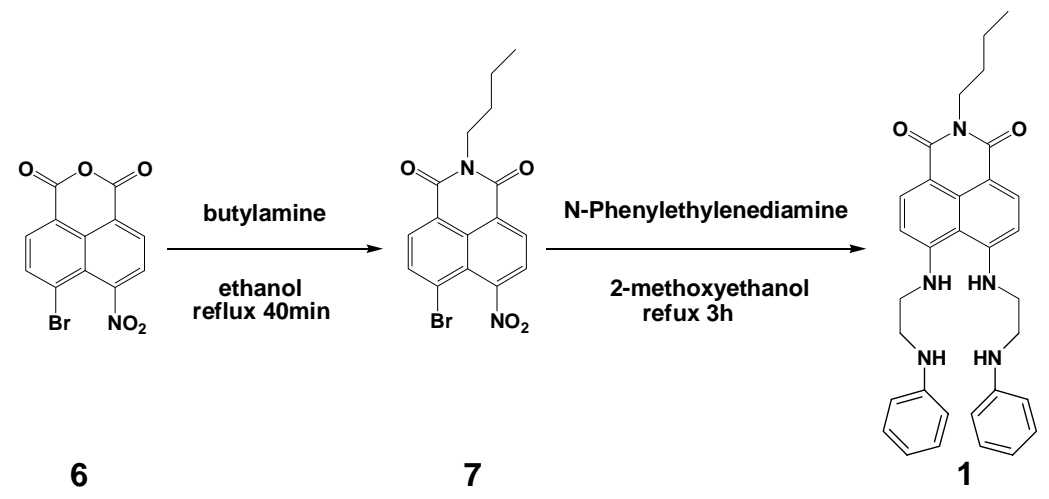

Compound $\mathbf{6}$ was synthesized according to the published procedures. ${ }^{\text {S2 }}$

\section{$\mathrm{N}$-butyl-4-bromo-5-nitro-1,8-naphthalimide (7).}

To a solution of $400 \mathrm{mg}$ (1.24 mmol) 4-bromo-5-nitro-1,8-naphthalic anhydride in 40 $\mathrm{mL}$ ethanol was added dropwise $90 \mathrm{mg}(1.24 \mathrm{mmol})$ butylamine in $12 \mathrm{~mL}$ ethanol. The mixture was then heated at reflux for $40 \mathrm{~min}$ and monitored by TLC. After the reaction was completed, the solvent was removed under reduced pressure. The crude product was then purified by chromatography on a silica gel column $\left(\mathrm{CHCl}_{3}\right)$ to give 7 as a white solid in $40 \%$ yield $(188 \mathrm{mg}) . \mathrm{Mp}: 175.8-176.2{ }^{\circ} \mathrm{C} .{ }^{1} \mathrm{H}-\mathrm{NMR}\left(\mathrm{CDCl}_{3}, 400 \mathrm{MHz}\right)$ $\delta 0.99(\mathrm{t}, J=7.2 \mathrm{~Hz}, 3 \mathrm{H}), 1.45(\mathrm{~m}, J=7.2 \mathrm{~Hz}, 2 \mathrm{H}), 1.71(\mathrm{~m}, J=7.2 \mathrm{~Hz}, 2 \mathrm{H}), 4.18(\mathrm{t}, J$ $=7.2 \mathrm{~Hz}, 2 \mathrm{H}), 7.93(\mathrm{~d}, J=8.0 \mathrm{~Hz}, 1 \mathrm{H}), 8.21(\mathrm{~d}, J=8.0 \mathrm{~Hz}, 1 \mathrm{H}), 8.51(\mathrm{~d}, J=8.0 \mathrm{~Hz}$, $1 \mathrm{H}), 8.71(\mathrm{~d}, J=8.0 \mathrm{~Hz}, 1 \mathrm{H}) .{ }^{13} \mathrm{C}-\mathrm{NMR}\left(\mathrm{CDCl}_{3}, 100 \mathrm{MHz}\right) \delta 13.95,20.49,30.21$, 40.92, 122.69, 123.72, 124.26, 125.99, 131.37, 132.49, 136.14, 162.21, 162.98. IR $\left(\mathrm{KBr}, \mathrm{cm}^{-1}\right): 3057,2963,2935,2912,2860,1706,1568,1541,1230,658,586 . \mathrm{HRMS}$ (EI) calcd for $\mathrm{C}_{16} \mathrm{H}_{13} \mathrm{BrN}_{2} \mathrm{O}_{4}\left[\mathrm{M}^{+}\right]$376.0059, found 376.0075 .

\section{$N$-butyl-4,5-di[2-(phenylamino)ethylamino]-1,8-naphthalimide (1).}

$0.3 \mathrm{~mL}(2.10 \mathrm{mmol}) \mathrm{N}$-Phenylethylenediamine was added dropwise to a solutuion of $100 \mathrm{mg}$ (0.266 mmol) $\mathrm{N}$-butyl-4-bromo-5-nitro-1,8-naphthalimide 7 in $3.00 \mathrm{~mL}$ 2-methoxyethanol, and then the mixture was heated to reflux for $3 \mathrm{~h}$ and monitored by TLC. After the reaction was completed, the solution was cooled at room temperature to give yellow needle crystals. The product was filtered off, washed with 2-methoxyethanol, and then dried in the air. The product was then purified by chromatography on a silica gel column $\left(\mathrm{CH}_{2} \mathrm{Cl}_{2} /\right.$ EtoAC, $\left.1: 1, \mathrm{v} / \mathrm{v}\right)$ to give $\mathbf{1}$ as a yellow crystal in $87 \%$ yield $(120 \mathrm{mg}) . \mathrm{Mp}: 203.5-204.1{ }^{\circ} \mathrm{C} .{ }^{1} \mathrm{H}-\mathrm{NMR}\left(\mathrm{CDCl}_{3}, 400 \mathrm{MHz}\right) \delta$ $0.94(\mathrm{t}, J=7.2 \mathrm{~Hz}, 3 \mathrm{H}), 1.41(\mathrm{~m}, J=7.2 \mathrm{~Hz}, 2 \mathrm{H}), 1.67(\mathrm{~m}, J=7.2 \mathrm{~Hz}, 2 \mathrm{H}), 3.37$ (s, 4H), 3.43(s, 4H), 4.10 (t, $J=7.2 \mathrm{~Hz}, 2 \mathrm{H}), 6.23(\mathrm{~s}, \mathrm{~N}-\mathrm{H}), 6.56(\mathrm{~d}, J=8.4 \mathrm{~Hz}, 2 \mathrm{H}), 6.67$ $(\mathrm{d}, J=7.2 \mathrm{~Hz}, 4 \mathrm{H}), 6.77(\mathrm{t}, J=7.6 \mathrm{~Hz}, 2 \mathrm{H}), 7.19(\mathrm{t}, J=7.6 \mathrm{~Hz}, 4 \mathrm{H}), 8.28(\mathrm{~d}, J=8.4 \mathrm{~Hz}$, $2 \mathrm{H}) .{ }^{13} \mathrm{C}-\mathrm{NMR}\left(\mathrm{CDCl}_{3}, 100 \mathrm{MHz}\right) \delta 14.06,20.67,30.61,40.03,43.47,44.04,107.25$, $112.04,112.75,113.59,119.01,129.80,132.26,133.62,147.89,152.21,164.66$. IR $\left(\mathrm{KBr}, \mathrm{cm}^{-1}\right): 3328,3058,2961,2934,1673,1626,1603,1513,1499,1310,996$. HRMS (EI) calcd for $\mathrm{C}_{32} \mathrm{H}_{35} \mathrm{~N}_{5} \mathrm{O}_{2}\left[\mathrm{M}^{+}\right]$521.2791, found 521.2779. 


\section{$N$-butyl-4-[2-(phenylamino)ethylamino]-1,8-naphthalimide (2).}

Compound 2 was prepared by standard methods ${ }^{\mathrm{S} 3}$ from 4-bromo-1,8-naphthalic anhydride by reaction with firstly butylamine and then $N$-Phenylethylenediamine.

Mp: $146.3-147.1{ }^{\circ} \mathrm{C} .{ }^{1} \mathrm{H}-\mathrm{NMR}\left(\mathrm{CDCl}_{3}, 400 \mathrm{MHz}\right) \delta 0.91(\mathrm{t}, J=7.2 \mathrm{~Hz}, 3 \mathrm{H}), 1.31(\mathrm{~m}, J$ $=7.2 \mathrm{~Hz}, 2 \mathrm{H}), 1.57(\mathrm{~m}, J=7.2 \mathrm{~Hz}, 2 \mathrm{H}), 3.40(\mathrm{~s}, 4 \mathrm{H}), 4.11(\mathrm{t}, J=7.2 \mathrm{~Hz}, 2 \mathrm{H}), 5.76(\mathrm{~s}$, $\mathrm{N}-\mathrm{H}), 6.53(\mathrm{t}, J=7.2 \mathrm{~Hz}, 1 \mathrm{H}), 6.61(\mathrm{~d}, J=8.0 \mathrm{~Hz}, 2 \mathrm{H}), 6.81(\mathrm{~d}, J=8.4 \mathrm{~Hz}, 1 \mathrm{H}), 7.07(\mathrm{t}$, $J=8.0 \mathrm{~Hz}, 2 \mathrm{H}), 7.67(\mathrm{t}, J=8.0 \mathrm{~Hz}, 1 \mathrm{H}), 8.24(\mathrm{~d}, J=8.4 \mathrm{~Hz}, 1 \mathrm{H}), 8.42(\mathrm{~d}, J=7.6 \mathrm{~Hz}$, $1 \mathrm{H}), 8.66(\mathrm{~d}, J=8.4 \mathrm{~Hz}, 1 \mathrm{H}) .{ }^{13} \mathrm{C}-\mathrm{NMR}\left(\mathrm{CDCl}_{3}, 100 \mathrm{MHz}\right) \delta 14.03,20.57,30.45$, 40.14, 42.88, 43.09, 104.44, 110.66, 113.50, 118.70, 120.36, 123.00, 124.83, 126.15, 129.60, 129.66, 131.16, 134.27, 147.78, 149.34, 164.20, 164.68. IR (KBr, cm $\left.{ }^{-1}\right): 3328$, 3043, 2953, 2869, 1673, 1633. HRMS (EI) calcd for $\mathrm{C}_{24} \mathrm{H}_{25} \mathrm{~N}_{3} \mathrm{O}_{2}\left[\mathrm{M}^{+}\right]$387.1947, found 387.2738.

Reference.

S1. X. Guo, X. Qian, L. Jia, J. Am. Chem. Soc. 2004, 126, 2272-2273.

S2. A. T. Peters, Y. S. S. Behesti, J. Soc. Dyers. Colour. 1989, 105, 29-35.

S3. M. S. Alexiou, V. Tychopoulos, S. Ghorbanian, J. H. P. Tyaman, R. G. Brown, P. I. Brittain, J. Chem. Soc. Perkin Trans. 2 1990, 837. 


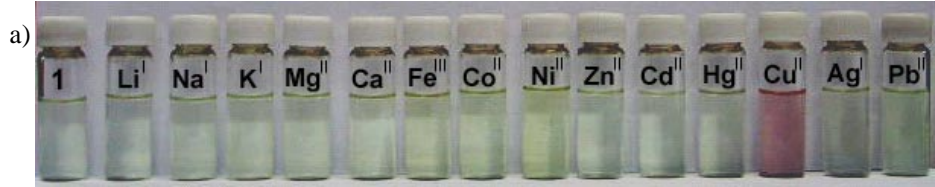

b)

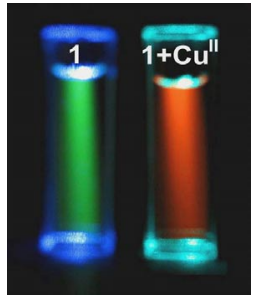

Figure S1. a) Color change of the ethanol-water (60:40, v/v, $50 \mathrm{mM}$ HEPES buffer, pH 7.2) solutions of compound $\mathbf{1}$ on addition of different metal ions. [1] $=10 \mu \mathrm{M}$, and the concentration of each metal ion was $50 \mu \mathrm{M}$. b) Visible emission observed from the solution of $\mathbf{1}$ and $\mathrm{Cu}(\mathrm{II})$. left, excited at $420 \mathrm{~nm}$; right, excited at $510 \mathrm{~nm}$.

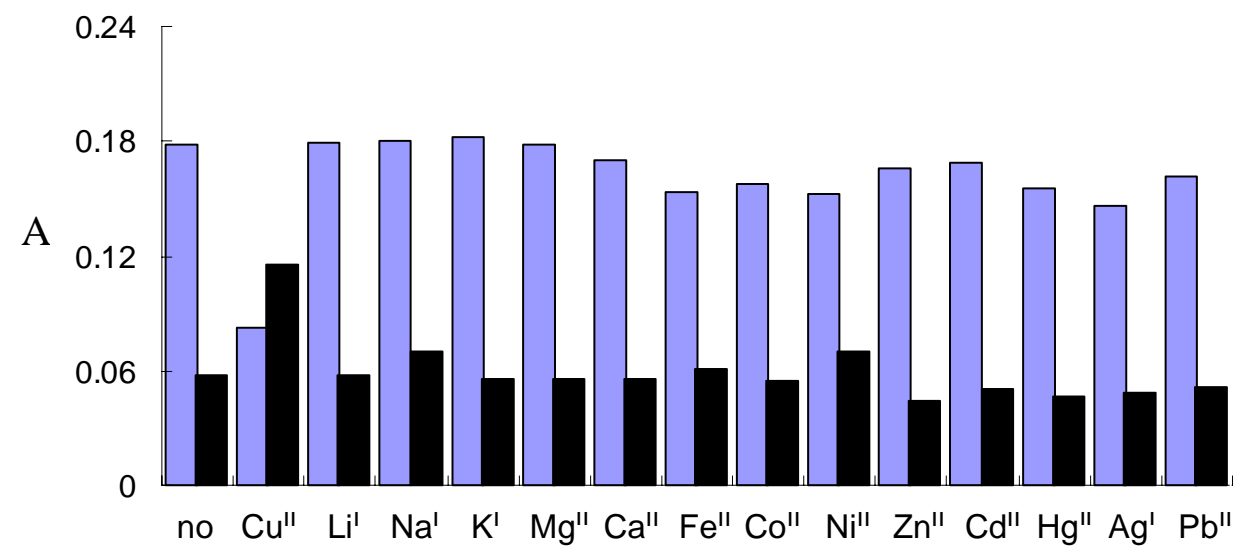

Figure S2. UV-Vis absorption response of 1 to various metal ions in ethanol-water solutions (60:40, $\mathrm{v} / \mathrm{v}, 50 \mathrm{mM}$ HEPES buffer, $\mathrm{pH}$ 7.2). [1] = $10 \mu \mathrm{M}$, and the concentration of each metal ion was 50 $\mu \mathrm{M}$. The blue (left) bars represent absorption at $419 \mathrm{~nm}$. The black (right) bars represent absorption at $509 \mathrm{~nm}$. 


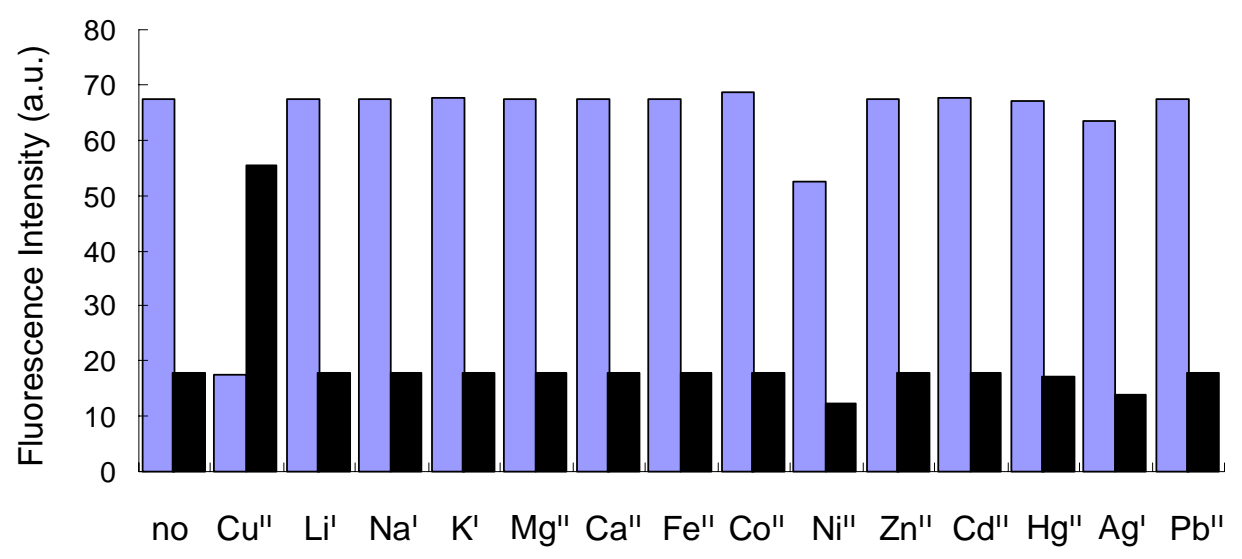

Figure S3. Fluorescence response of 1 to various metal ions in ethanol-water solutions (60:40, v/v, $50 \mathrm{mM}$ HEPES buffer, $\mathrm{pH} 7.2$ ). [1] $=10 \mu \mathrm{M}$, and the concentration of each metal ion was $50 \mu \mathrm{M}$. The blue (left) bars represent emission at $518 \mathrm{~nm}$ (excited at $420 \mathrm{~nm}$ ), The black (right) bars represent emission at $592 \mathrm{~nm}$ (excited at $510 \mathrm{~nm}$ ). 